\title{
Experimental Investigation on Road Vehicle Active Suspension
}

\author{
Prabu Krishnasamy* - Jancirani Jayaraj-Dennie John \\ Anna University, Department of Automobile Engineering, India
}

This paper presents an investigation report for an electronically controlled pneumatic suspension system. The performance improvement in the passenger's comfort and attitude behaviour are evaluated for a proportional integral derivative (PID) controlled pneumatic suspension design. An appropriate mathematical model is developed for a single wheel suspension with the passenger seat system. The simulation is accomplished through LABVIEW and lab-based experimental analysis is conducted. Based on simulation and experimental values, the enhanced performance is shown through comparative results. The proposed system with the PID control improves the ride comfort and provides better road-holding characteristics, as compared to the passive suspension system.

Keywords: PID, actuator, pneumatic suspension, active control, refined Zi-Ni control

\section{O INTRODUCTION}

The primary function of the vehicle suspension system is to provide vehicle body isolation from road irregularities, to maximise passenger ride comfort and retain continuous contact between the tyre and road surface. The ability of the suspension to filter out vibrations on an uneven road surface determines ride comfort [1]. Concerning the suspension system, good ride comfort is offered by soft suspension, whereas a stiff suspension is required for suddenly applied loads. A good suspension requires a trade-off between these two parameters. In a passive suspension, the suspension spring stiffness and damping values are predefined. In a semi-active suspension, the system can be controlled only in one direction: opposite to the velocity of the damper extension. In contrast, an actively controlled pneumatic suspension system adapts according to changing road conditions by continuously varying its stiffness value, and produces better sprung mass isolation. Vehicle suspension has been extensively studied for quarter-car models with two-mass systems [2] to [4]. For this study, a three mass, single-wheel suspension system with a passenger seat is considered; the masses are the passenger seat with a passenger, the vehicle body and the axle assembly [5].

The control algorithms are usually based on linear quadratic, fuzzy logic, linear quadratic regulator, linear quadratic Gaussian and a proportional integral derivative (PID) controller based on feedback control approaches. Some of the researchers preferred a PID controller, which is used with a simple control algorithm, and a robust and efficient control [6]. Therefore, the pneumatic spring stiffness controlled by the PID algorithm has been adopted for this study. From previous research, it is found that the PID control for the pneumatic suspension system can improve the occupant's comfort [7] and [8]. Simulation and an experimental study based on onequarter T suspensions with PID controllers are utilized [9] and [10] in this paper. This study focuses on the compromise of the comfort and road holding of the vehicle by the influence of the pneumatic actuator and examines the performance of the suspension system through simulation and experiment.

The paper has the following sections: Section 1 introduces the need for a pneumatic suspension system and their control methods. A system is proposed based on the information gathered from the literature review that has been carried out in this area. In Section 2, the assumptions made to develop the mathematical model and the simulation model of the pneumatic suspension system are presented. The control algorithm, controller parameters, effect of control parameters on the system performance and controller design are illustrated in Section 3. The road bump model, simulation, experimental methods and its results are presented in Section 4. The simulation and testing results of the actively controlled pneumatic seat suspension system are compared with the passive suspension system.

\section{PNEUMATIC SUSPENSION DYNAMICS}

The dynamic model of the proposed system is developed according to the following assumptions: passenger seat, vehicle body and axle assembly are both considered as a rigid body; the tyre is considered as a linear spring with equivalent stiffness and damping force of the tyre is neglected. Passive suspension is considered to be a spring and vibration damper in parallel connection; additionally, an active pneumatic spring of varying stiffness is connected, which is used as a force actuator. This spring allows for dynamic variations in stiffness characteristics 
while the vehicle is in motion. Vehicle passenger mass with the seat mass is taken as $m_{p s}$. The sprung mass which represents a portion of the total mass of the vehicle is $\mathrm{m}_{\mathrm{s}}$ and the un-sprung mass $m_{u s}$ representing one of the wheel and axle. The radial tyre stiffness and suspension spring stiffness are represented by $k_{t}$ and $k_{s}$, respectively. The damping coefficient of the damper is represented by $c_{s}$, and $k_{c s}$ represents the stiffness of the pneumatic spring. The input $x_{r}$ is assumed to be the road bump, $x_{p s}, x_{s}$ and $x_{u s}$ are the vertical displacement of the passenger seat, sprung and un-sprung mass, respectively. Based on Newton's second law, the dynamic equation of motion for a single wheel suspension system is obtained using the above assumptions.



Fig. 1. Pneumatic suspension system

The pneumatic vehicle suspension model is shown in Fig. 1. The inputs are the random road surface and pneumatic actuator force Pneumatic actuator force $\left(F_{b}\right)$ is the product of the relative displacement of the sprung, un-sprung mass and the dynamic stiffness of the pneumatic system $\left(k_{c s}\right)$, which acts on the vehicle body. The equation of motion for a pneumatic suspension model is represented by the following differential equations, Eqs. (1) to (3).

$$
\begin{gathered}
m_{p s} \ddot{x}_{p s}+c_{p s}\left(\dot{x}_{p s}-\dot{x}_{s}\right)+k_{p s}\left(x_{p s}-x_{s}\right)=0, \\
m_{s} \ddot{x}_{s}+c_{p s}\left(\dot{x}_{s}-\dot{x}_{p s}\right)+k_{p s}\left(x_{s}-x_{p s}\right)+ \\
+c_{s}\left(\dot{x}_{s}-\dot{x}_{u s}\right)+k_{s}\left(x_{s}-x_{u s}\right)-k_{c s}\left(x_{s}-x_{u s}\right)=0, \\
m_{u s} \ddot{x}_{u s}+k_{t}\left(x_{u s}-x_{r}\right)+c_{s}\left(\dot{x}_{u s}-\dot{x}_{s}\right)+ \\
+k_{s}\left(x_{u s}-x_{s}\right)+k_{c s}\left(x_{s}-x_{u s}\right)=0 .
\end{gathered}
$$

Choose state space output vector:

$$
\begin{gathered}
x^{T}=\left[\begin{array}{l}
x_{p s} \dot{x}_{p s} x_{s} \dot{x}_{s} x_{u s} \dot{x}_{u s}
\end{array}\right], \\
y^{T}=\left[\ddot{x}_{p s} \ddot{x}_{s} x_{p s}-x_{s} x_{s}-x_{u s} x_{u s}-x_{r}\right] .
\end{gathered}
$$

State space suspension model:

$$
\begin{aligned}
& \dot{x}=A x+B u, \\
& y=C x+D u,
\end{aligned}
$$

$$
A=\left[\begin{array}{cccccc}
0 & 1 & 0 & 0 & 0 & 0 \\
-\frac{k_{p s}}{m_{p s}} & -\frac{c_{p s}}{m_{p s}} & \frac{k_{p s}}{m_{p s}} & \frac{c_{p s}}{m_{p s}} & 0 & 0 \\
0 & 0 & 0 & 1 & 0 & 0 \\
\frac{k_{p s}}{m_{s}} & \frac{c_{p s}}{m_{s}} & \frac{-\left(k_{p s}+k_{s}+k_{c s}\right)}{m_{s}} & -\frac{\left(c_{p s}+c_{s}\right)}{m_{u s}} & \frac{k_{s}+k_{c s}}{m_{s}} & \frac{c_{s}}{m_{s}} \\
0 & 0 & 0 & 0 & 0 & 1 \\
0 & 0 & \frac{k_{s}+k_{c s}}{m_{u s}} & \frac{c_{s}}{m_{u s}} & \frac{-\left(k_{s}+k_{c s}+k_{t}\right)}{m_{u s}} & -\frac{c_{s}}{m_{u s}}
\end{array}\right],
$$

$$
B=\left[\begin{array}{c}
0 \\
0 \\
0 \\
0 \\
0 \\
\frac{k_{t}}{m_{u s}}
\end{array}\right], \quad C=\left[\begin{array}{llllll}
1 & 0 & 0 & 0 & 0 & 0
\end{array}\right] .
$$

The suspension travel is the relative displacement of the vehicle body and the axle assembly. The differences of un-sprung mass displacement and road height is the tyre displacement. In the air spring dynamics the force $\left(F_{b}\right)$ is the total pressure ( multiplied with the area $\left(A_{e}\right)$. As a result, pressure increases with displacement. Based on the above statement, the dynamic stiffness Eq. (9) is derived where the reservoir pressure $\left(P_{r}\right)$, gauge pressure $\left(P_{g}\right)$ and atmospheric pressure $\left(P_{a}\right)$ are taken into account. Therefore the static stiffness of the pneumatic spring is given by Eq. (8)

$$
k_{c s}=\left(P_{r}+P_{a}+P_{g}\right) \frac{A_{e}^{2}}{V}+P_{g} \frac{d A_{e}}{\mathrm{dy}} .
$$

In the dynamic condition, the change in effective area with deflection is extremely small i.e., constant effective area, the dynamic stiffness of the pneumatic system is:

$$
k_{c s, d \text {,ymanic }}=\gamma\left(P_{r}+P_{a}+P_{g}\right) \frac{A_{e}^{2}}{V} .
$$




\section{PID CONTROLLER}

The performance of the suspension system can be optimised by a proper design and implementation of the controller. The PID feedback controller is used, which is essential to maintain the position of the passenger seat and car body under equilibrium. The function of the controller is to minimise the difference between the set point level and the measured value. The proportional gain $\left(K_{P}\right)$, integral gain $K_{i}$ and derivative gain $K_{d}$ are the parameters which influence the controller design. The Ziegler-Nichols and refined Ziegler- Nichols (RZN) tuning methods are considered while designing the PID controller. The performance of the controller is enhanced through proper selection of the PID control parameters which brings down the rattle space of the system, the acceleration of the passenger seat of the vehicle body and improves the tyre-road contact. The ability of the PID controller for the pneumatic suspension system is evaluated with a simulation of the passive suspension system.

Ziegler and Nichols [11] described a method for tuning the parameters of PID controller. There are two methods in Ziegler-Nichols: the closed loop method and open loop method. The present paper implements the closed loop control method in a pneumatic suspension system. The closed loop method applies a time delay constant.
The Ziegler-Nichols tuning method is mathematically represented in Eq. 10.

$$
K_{p} e+K_{i} \int e d t+K_{p}\left(\frac{d e}{d t}\right) .
$$

\subsection{Refined Ziegler- Nichols (RZN)}

The refined Ziegler Nichols settings are introduced by adding a new parameter $\beta$ [11] to [13] in the proportional action of the controller. The new parameter $\beta$ can reduce the overshoot to acceptable levels, and thus gives a good set point response. When the $\beta$ value is less than unity, the control mathematical equation of the proposed controller is expressed [12] as:

$$
\begin{aligned}
u_{t}(s) & =K_{p}\left(\beta e(t)+1 / T_{i} \int_{0}^{t} e(\tau) d \tau\right)- \\
& -K_{p}\left((1-\beta) y+T_{d} \frac{d y}{d t}\right) .
\end{aligned}
$$

\section{SIMULATION AND EXPERIMENT}

The suspension plant model is developed using motion equations. The simulation model, shown in Fig. 2, is aided with LABVIEW software and the road profile is modelled as a mathematical cosine function in Eq.



Fig. 2. Simulation pneumatic suspension model 
(12) to investigate the performance of the pneumatic suspension under general road conditions. A single bump is given as road input for which the dynamic response of a single wheel is investigated.

In this study, a typical road disturbance is used and it is mathematically represented as:

$$
x_{r}(t)=\left\{\begin{array}{cc}
a[1-\cos 8 \pi t] & (0.5 \leq t<0.75) \\
0 & \text { otherwise }
\end{array},\right.
$$

where $a$ denotes the bump amplitude. The single bump input, with height of $0.05 \mathrm{~m}$ is obtained by setting $a=$ 0.025 [13] and [14], and is used to study the system response. Furthermore, the maximum travel distance of the suspension travel is aimed to be limited within $\pm 8 \mathrm{~cm}$.

The mathematical model of the system defined in Eqs. (6) and (7) and the RZN proportional integral derivative controller in Eq. (11) were simulated, see Fig 2. The performance of the passive system is compared to the PID-controlled pneumatic suspension system. The simulation parameters value based on the laboratory test bench [11] are as follows: passenger, sprung and un-sprung mass plates are 5.25, 18.2 and $7.25 \mathrm{~kg}$, respectively. Suspension stiffness and radial tyre stiffness are 1570 and $4850 \mathrm{~N} / \mathrm{m}$. The damping value of the damper is $100 \mathrm{Ns} / \mathrm{m}$.

An experimental test rig is used to analyse the performance of the pneumatic suspension system, which is shown in Fig. 8. The main structure of the test bench comprises a steel frame with two vertical guide rods to guide the mass plates through the linear bearing. A set of coil springs is placed at the appropriate locations to provide suspension and tyre stiffness. The $0.05 \mathrm{~m}$ excitation is generated by the cam, powered by speed reduction gear box along with motor setup.

In order to accomplish the design objective of an active suspension system i.e. to increase the ride comfort and road holding, there are the three parameters to be observed in simulations and experiments: the passenger acceleration, car body acceleration and the suspension deflection. From the observation, the descending value of the peak acceleration of the passenger seat is recorded by the uncontrolled passive system, experiment and simulation. This evidently shows that RZN control offers the better result in the passenger seat acceleration and travel as shown in Figs. 3 and 4. Fig. 4. Illustrates that the proposed system can effectively absorb the vehicle vibration in comparisons to the PID method and the passive system. The body acceleration, shown in Fig. 5, in the PID design system is reduced

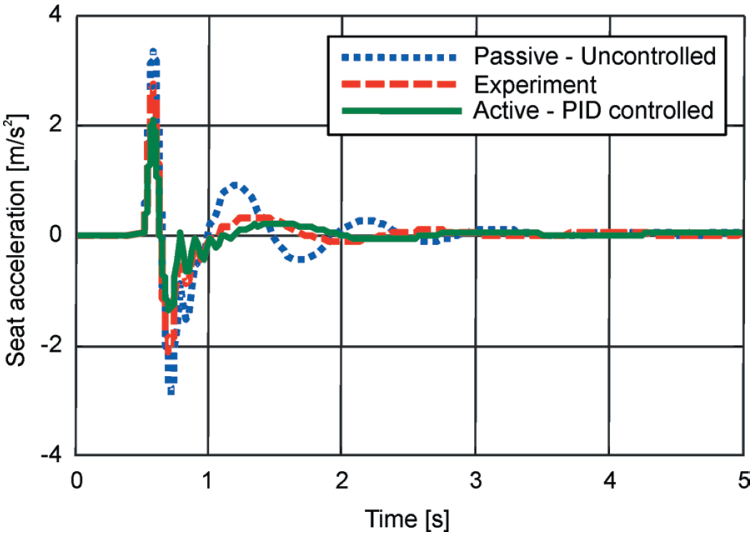

Fig. 3. Results showing the seat acceleration for $5 \mathrm{~cm}$ bump road input



Fig. 4. Results showing the seat travel for $5 \mathrm{~cm}$ bump road input



Fig. 5. Results showing the body accelerations for $5 \mathrm{~cm}$ bump road input

significantly, which guarantees better ride comfort. The suspension travels (see Fig. 6) of a controlled active suspension system and a uncontrolled passive suspension system are used for comparison purposes. The result shows that the suspension travel is limited within the travel limit i.e. $\pm 8 \mathrm{~cm}$. The highest 


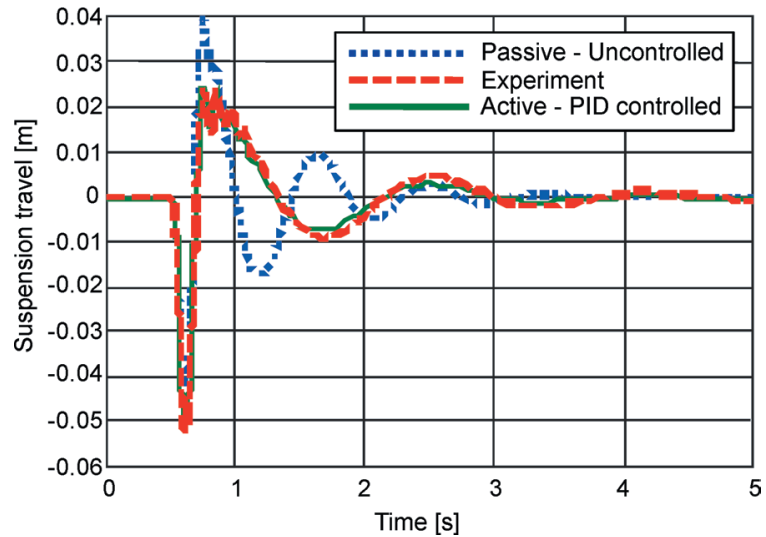

Fig. 6. Results showing the suspension travel for $5 \mathrm{~cm}$ bump road input

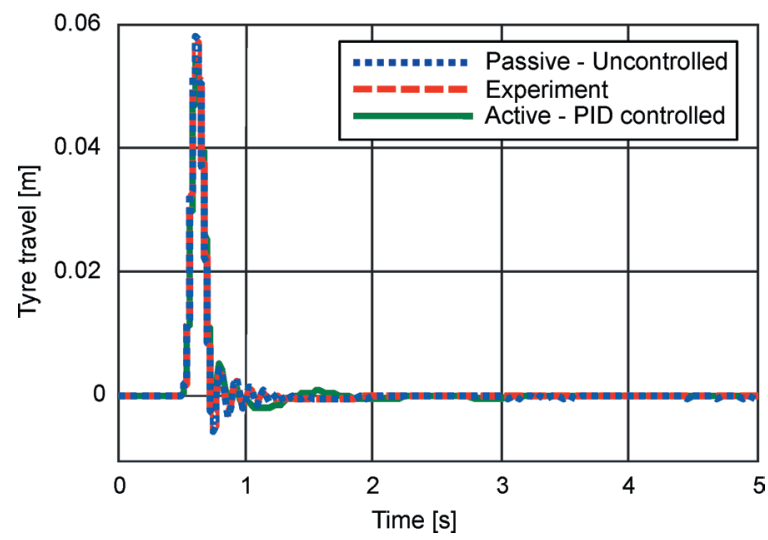

Fig. 7. Results showing the tyre travel for $5 \mathrm{~cm}$ bump road input

Table 1. Quantitative result with passive system comparison of peak values

\begin{tabular}{lccc}
\hline Parameters & Passive & Simulation & Experiment \\
\hline Seat acceleration $\left[\mathrm{m} / \mathrm{s}^{2}\right]$ & 3.3194 & 2.0782 & 2.7085 \\
\hline Body acceleration $\left[\mathrm{m} / \mathrm{s}^{2}\right]$ & 4.0721 & 2.0855 & 2.3998 \\
\hline Seat travel $[\mathrm{m}]$ & 0.0215 & 0.011 & 0.0021 \\
\hline Suspension travel $[\mathrm{m}]$ & 0.0388 & 0.0237 & 0.0245 \\
\hline Tyre travel $[\mathrm{m}]$ & 0.0543 & 0.0537 & 0.0576 \\
\hline
\end{tabular}

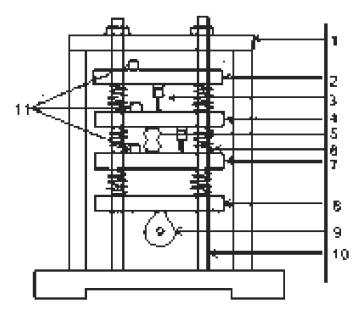

Fig. 8. Experimental setup; 1. steel base structure, 2. passenger mass, 3. seat damper, 4. sprung mass, 5. pneumatic actuator, 6. coil spring, 7. unsprung mass, 8. guide plate, 9. disturbance actuator, 10. guide rods, 11 . accelerometer sensors suspension travel is recorded by the passive system, shown in Fig. 6, and the RZN PID performs better as compared to the other one. Tyre travel, shown in Fig. 7 , is noticeably small in simulation and slightly raised in the experimental setup for the proposed controller.

In the case of body acceleration, it is observed that the lowest possible acceleration is made by RZN and the maximum by the passive system (Table 1).

\section{CONCLUSION}

From the results obtained, the RZN control algorithm produces the lowest accelerations at the passenger seat. Considering the simulation and test results, the RZN control algorithm is effective and reliable for pneumatic suspension systems. All other parameters are found to be within satisfactory limits. Although the peak tyre travel is increased, it lasts for a very short duration in relation to the passive system. This results in improved road holding characteristics when the vehicle is negotiating the bump. Therefore, it is concluded that the active pneumatic suspension system with the PID controller improves ride comfort while retaining road holding characteristics, as compared to the passive suspension system. Thus, the results are also confirmed if the study has extended to examine the behaviour at different road amplitudes.

\section{REFERENCE}

[1] Genta, G., Morello, L. (2009). The Automotive Chassis, Vol. 2: System Design, Mechanical Engineering Series, Springer, DOI:10.1007/978-1-4020-8675-5.

[2] Karnoop, D. (1986). Theoretical limitations in active vehicle suspension. International Journal of Vehicle Mechanics and Mobility, vol. 15, no. 1, p. 41-54, DOI:10.1080/00423118608968839.

[3] Herdrick, J.K., Batsuen, T. (1990). Invariant properties of automotive suspension. Proceedings of the institution of mechanical engineers, Part D: Journal of Automobile Engineering, vol. 204, no. 1, p. 21-27, DOI:10.1243/PIME_PROC_1990_204_128_0.

[4] Hrovat, D. (1993). Application of optimal control to advance automotive suspension design. Transaction of ASME, Journal of Dynamics Systems, Measurement And Control, vol. 115, p. 328-342, DOI: DOI:10.1115/1.2899073.

[5] Rakheja, S., Afework, Y., Sankar, S. (1994). An analytical and experimental investigation of driver seat suspension system. Vehicle System Dynamics: International Journal of Vehicle Mechanics and Mobility, vol. 23, no. 1, p. 501-524, DOI:10.1080/00423119408969072.

[6] Li, M., Li, Z., Shen, X., Guo, J. (2010). Study on PID control for semi active air suspension for riding 
comfort. Second WRI Global Congress on Intelligent System, p. 47-50, DOI:10.1109/GCIS.2010.82.

[7] Toyofuku, K., Yamada, C., Kagawa, T. (1999). Study on dynamic characteristics analyses of air spring with auxiliary chamber. JSAE Review, vol. 20, no. 3, p. 349355, DOI:10.1016/S0389-4304(99)00032-6.

[8] Pooya, M., Reza, K. (2008). Active stiffness and damping control of air mounted system. ASME International Mechanical Engineering Congress and Exposition, Volume 11: Mechanical Systems and Control, p. 531-544, DOI:10.1115/IMECE2008-66272.

[9] Renn, J-C., Wu, T.H. (2007). Modeling and control of new $1 / 4 \mathrm{~T}$ servo hydraulic vehicle active suspension system. Journal of Marine Science and Technology, vol. 15 , no. 3, p. 265-272.

[10] Koch, G., Pellegrini, E., Spirk, S., Lohmann, B. (2010). Design and modeling of a quarter car vehicle test rig for active suspension control. Technical Reports on Automatic Control, vol. TRAC-5, p.1-28.

[11] Ziegler, J.G., Nichols N.B. (1942). Optimum settings for automatic controllers. Transactions of the ASME, vol. 64, p. 759-768.

[12] Astrom, K.J., Hagglund, T. (2004). Revisiting the Ziegler-Nichols step response method for PID control. Journal of Process Control, vol. 14, no. 6, p. 635-650, DOI:10.1016/j.jprocont.2004.01.002.

[13] Krishnasamy, P., Jayaraj, J. (2012). Vehicle active suspension system simulation with optimum PID technique. Wulfenia Journal, vol. 19, no. 9, p. 119-121.

[14] Lin, J.S., Kanellakopoulos, I. (1997). Nonlinear Design of Active Suspension. IEEE Control System Magazine, vol. 17 , no. 3, p. 45-59, DOI:10.1109/37.588129. 